Journal of Biomedical

Materials Research

WILEY

Part B: Applied Biomaterials

\title{
Wear effects on microscopic morphology and hyaluronan uptake in siloxane-hydrogel contact lenses
}

\begin{tabular}{|r|l|}
\hline Journal: & Journal of Biomedical Materials Research: Part B - Applied Biomaterials \\
\hline Manuscript ID: & JBMR-B-14-0150.R1 \\
\hline Wiley - Manuscript type: & Original Research Report \\
\hline Complete List of Authors: & $\begin{array}{l}\text { O4-Jun-2014 } \\
\text { tavazzi, Silvia; University of Milano Bicocca, Materials Science Department } \\
\text { Fagnola, Matteo } \\
\text { Cozza, Federica } \\
\text { Ferraro, Lorenzo; University of Milano Bicocca, MaterialsScience } \\
\text { Department } \\
\text { Borghesi, Alessandro; University of Milano Bicocca, Materials Science } \\
\text { Department } \\
\text { Ascagni, Miriam; University of Milan, Interdepartmental Center of } \\
\text { Advanced Microscopy, CIMA, } \\
\text { Farris, Stefano; University of Milan, DeFENS, Department of Food, } \\
\text { Environmental and Nutritional Sciences }\end{array}$ \\
\hline \hline Keywords: & \begin{tabular}{l} 
contact lens, wear, SEM techniques, hydrogel, surface modification \\
\hline
\end{tabular} \\
\hline
\end{tabular}




\title{
Wear effects on microscopic morphology and hyaluronan uptake
} in siloxane-hydrogel contact lenses

\author{
Silvia Tavazzi*, Martina Tonveronachi, Matteo Fagnola, Federica Cozza, Lorenzo Ferraro, \\ Alessandro Borghesi, Miriam Ascagni, and Stefano Farris
}

\begin{abstract}
Dr Silvia Tavazzi, Martina Tonveronachi, Matteo Fagnola, Federica Cozza, Lorenzo Ferraro, Prof. Alessandro Borghesi

Materials Science Department, University of Milano Bicocca, via Cozzi 55, I-20125 Milan, Italy. Fax: +390264485400; Tel: +390264485035; E-mail: silvia.tavazzi@unimib.it

Dr Miriam Ascagni

Interdepartmental Center of Advanced Microscopy, CIMA, University of Milan, via Celoria 26, I20133, Milan, Italy
\end{abstract}

Dr Stefano Farris

DeFENS, Department of Food, Environmental and Nutritional Sciences, Packaging DivisionUniversity of Milan, Via Celoria 2, I-20133, Milan, Italy

* University of Milano Bicocca, Materials Science Department

Via r. Cozzi 55, I-20125 Milan (Italy)

Phone: +3902 64485035, Fax: +390264485400

E-mail: silvia.tavazzi@unimib.it

Running Heads: Wear-dependent morphology of contact lenses 


\begin{abstract}
The purpose of this study was a comparison between new and worn siloxane-hydrogel contact lenses in terms of microscopic structure, surface morphology, and loading of hyaluronan. The analyses were performed by scanning electron microscopy, with the support of the freeze-drying technique, and by fluorescence confocal microscopy. Along the depth profile of new lenses, a thin porous top layer was observed, which corresponds to the region of hyaluronan penetration inside well defined channels. The time evolution was followed from one day to two weeks of daily wear, when a completely different scenario was found. Clear experimental evidence of a buggy surface was observed with several crests and regions of swelling, which could be filled by the hyaluronan solution. The modifications are attributed to the progressive relaxation of the structure of the polymeric network.
\end{abstract}

\title{
KEYWORDS:
}

- Contact lens

- Siloxane-hydrogel

- Freeze-drying

- Scanning electron microscopy

- Hyaluronan 


\section{Introduction}

In recent applications, disposable contact lenses are often made of siloxane-hydrogel materials. Due to the interaction with the tears and with the cornea, their morphological properties, porosity, and hydrophilicity are expected to influence the clinical performances and the comfort during wear. Moreover, in recent years, the lens absorption of various molecules and macromolecules has been taken into consideration. One reason is the presence of molecules and macromolecules both in tears and in maintenance solutions. Another reason is the interest towards contact lenses for uptake and release of drugs and macromolecules of special interest. ${ }^{[1-3]}$ Hyaluronan (also called hyaluronic acid, HA) is one of these molecular species of special interest for its ophthalmic advantages. It is a polysaccharide present in many tissues of the human body, such as connective, epithelial, and neural tissues, with applications in medicine, pharmacy, biotechnology, and ophthalmology, for example as a remedy for the dry-eye syndrome. ${ }^{[4]}$ Moreover, the HA uptake in contact lenses plays a role to improve the comfort and also to prevent the adsorption in the polymeric network of the contact lens of other substances, which could possibly determine problems during the wear, such as cytotoxicity or ocular irritation. ${ }^{[5-12]}$ HA can also be considered a model system to discuss the loading capability of the lenses both before and after wear and to investigate the loading mechanisms in disposable contact lenses of ocular drugs, as potential controlled-release delivery systems. ${ }^{[13-19]}$

To determine the amount of HA loaded in one lens after exposure to a water HA solution, some experimental methods were presented based on the use of different dyes, which form complexes with the HA. ${ }^{[18-21]}$ These methods are indirect because the dye forms complexes also with the polymeric material of the lens itself, so that the content of HA in each lens was deduced from the variation of the HA content in the loading solution before and after the procedure. However, the methods based on dyes are typically time-consuming and they require many steps. Other limitations of these techniques are the lack of information on the HA distribution and penetration in the core of the lens, as well as on the morphology and structure of the HA-loaded lenses, such as the presence 
of pores, the capability of the HA to fill them, the possible aggregation of HA in clusters, the size of these clusters, and the compatibility with their internalization in the polymeric network of the lens. As far as the lens structure is concerned, few studies performed by scanning electron microscopy are reported in the literature, mainly on unworn contact lenses, ${ }^{[22-25]}$ but further details deserve to be investigated, such as the effects of wear and the relationships with solute uptake. Detailed investigations also require the improvement of the acquisition technique.

The focus of the present work is the comparison between new and worn contact lenses in terms of microscopic surface morphology, structure, porosity, HA distribution, and penetration depth inside the lens. To this scope, a combined study was performed by scanning electron microscopy (SEM) and confocal fluorescence microscopy, the former after freeze-drying the samples to perform SEM analyses on dehydrated lenses by maintaining the structure of the hydrated lens. A siloxanehydrogel contact lens was used as model system.

\section{Experimental}

Fluorescein-labelled HA (F-HA) with molecular weight $1000 \mathrm{kDa}$ was purchased from TdB Consultancy (Sweden). Aqueous salt solution containing sodium chloride $(0.9 \%)$ was purchased from Laboratoires Alcon (France). F-HA was dissolved in the salt solution with F-HA concentration $0.2 \% \mathrm{w} / \mathrm{v}$. Siloxane-hydrogel F-HA-free contact lenses of the I FDA group $(-3.00 \mathrm{D}$, thickness at the centre $\sim 90 \mu \mathrm{m}$, hydration $\sim 50 \%$ ) were supplied by Safilens (Italy). They were loaded with F-HA by immersion at room temperature in the F-HA salt solution.

Both before and after the loading procedure, scanning electron microscopy (SEM) micrographs were obtained using a LEO 1430 (Carl Zeiss, Oberkochen, Germany) operating at $20 \mathrm{kV}$ and $3 \cdot 10^{-3}$ Torr. Before analyses, samples were firstly freeze dried $\left(-55^{\circ} \mathrm{C}, 0.63 \mathrm{mbar} \times 24\right.$ hours $)$ using an ALPHA 1-2 LDplus freeze dryer (Martin Christ, Osterode am Harz, Germany). Then, a film of gold was sputtered on the lens surface with thickness of approximately $10 \mathrm{~nm}$ (to avoid charging the 
samples) using a Semprep 2 sputter coater (Nanotech Ltd., Prestwick, UK) at $10 \mathrm{~mA}$.

Fluorescence confocal micrographs were obtained using the Leica TCSNT confocal laser scanning microscope (Leica Microsystems, Wetzlar, Germany). For each lens a Z-stack was acquired (an image every $0.5 \mu \mathrm{m}$ ) to correctly evaluate the penetration depth of F-HA. The start point of the Zstack was fixed at the plane where the green fluorescence appeared and the end point was fixed at the plane where the green fluorescence disappeared.

\section{Results and discussion}

Figure 1 shows two typical SEM micrographs taken from the surface of HA-free contact lenses. Both empty pores (with diameters as large as several micrometers) and a fibrous texture of the material are clearly detected in Fig. 1a. Smaller pores with diameters of the order of 1-2 $\mu \mathrm{m}$ in size are detected in Fig. 1b. These different morphologies at the microscopic scale were observed also by analyzing different areas of the same lens, thus revealing a heterogeneous pattern attributable to the manufacturing process. According to the Lowman definition, ${ }^{[20,26]}$ this siloxane-hydrogel (at least the volume close to the lens surface observed in Fig. 1) can be reasonably defined as an intermediate case between the so-called macro-porous hydrogels, with typical pores in the range 0.1 $-1 \mu \mathrm{m}$, and the so- called super-porous hydrogels (tens and hundreds of $\mu \mathrm{m}$ ).

SEM analyses after the HA loading yielded a different scenario, as confirmed by the representative micrographs in Fig. 2. Pores, when present, were found to be much less pronounced. The difference can be attributed to the filling of the pores by HA. Another SEM image of a HA loaded lens is reported in Fig. 3. The different scale compared to Fig. 2 allows capturing a new branched morphology and a thickening of the main walls of the pores, which were attributed to the internalized HA, similarly as the morphological differences between the images of Figs. 2 and 1. For comparison, we mention that the surface properties of the same type of contact lenses loaded with $800 \mathrm{kDa} \mathrm{HA}$ were also investigated by atomic force microscopy (AFM), as reported 
elsewhere. ${ }^{[27]}$ The AFM micrographs were taken on sample areas of $(10 \times 10) \mu \mathrm{m}^{2}$ and $(5 \times 5) \mu \mathrm{m}^{2}$, which are lower than the areas here investigated. By AFM, a relatively low surface roughness was measured of approximately $2-3 \mathrm{~nm}$ on the area of $(10 \times 10) \mu \mathrm{m}^{2}$. No depressions of the surface due to the presence of deep pores were detected on unworn lenses. The lack of pores agrees with the filling of the pores of the lens with HA, as here observed by SEM. However, since the contact lenses were not freeze-dried before the AFM analyses, we cannot exclude that the lack of pores was due to their intrinsic disappearance owing to the water evaporation from the lens during the AFM analysis.

By SEM analysis, it was also possible investigating the distribution of the pores inside the lens along its depth profile. Figure 4 shows a SEM micrograph of the depth profile of a contact lens. A top porous layer can be clearly distinguished from the bulk. A further confirmation of the presence of a porous thin layer was obtained by fluorescence microscopy. Figure 5 shows two exemplificative fluorescence micrographs acquired from the surface of F-HA loaded lenses. The depth profile (i.e. the extent of the penetration of F-HA across the thickness of the lens) is visualized at the bottom and right sides of the main surface micrographs. Spots of emitted light from the surface are clearly seen, attributed to the F-HA fluorescein emission. By analyzing many lenses, emission was typically found to originate from channels with depth of about 5-10 $\mu \mathrm{m}$, as for example in the case reported in Fig. 5a. This value is much lower than the thickness of the lens, which is about $90 \mu \mathrm{m}$ thick at the centre and thicker in the periphery. This result is attributed to the reduced depth of the lens pores, which prevents the internalization of F-HA to the core. However, in few cases emission was also observed from deeper channels (of the order of tens of $\mu \mathrm{m}$ ). For example, in the case reported in Fig. $5 \mathrm{~b}$ the emission was detected by scanning the sample from the surface to a depth of about $100 \mu \mathrm{m}$, a value which is close to the thickness of the lens in the peripheral region, where the micrograph was taken. Noteworthy, the emitting spots have approximately the same size and the same surface density as the structures observed by SEM, which 
can thus be definitely attributed to pores filled with F-HA. Based on the fluorescence microscopy results, this siloxane-hydrogel can be classified as an intermediate case between a macro-porous hydrogel and a super-porous hydrogel, only as far as the layer close to the lens surface is concerned, within a variable depth from few micrometers to tens of $\mu \mathrm{m}$. The bulk of the lens is likely to belong to the micro-porous or non-porous hydrogel groups, based on the Lowman definition. ${ }^{[20,26]}$

For an approximated evaluation of the content of F-HA per lens, the fluorescence micrographs were analyzed to obtain quantitative information on the emitting volume. Figure 6 shows the statistical frequency distribution of the area of the fluorescent spots, as deduced from Fig. 5a. Since the micrograph resolution was $0.3 \mu \mathrm{m} / \mathrm{pixel}$, emission spots with area lower than $0.6 \mu \mathrm{m}^{2}$ (i.e. with radius lower than about $0.44 \mu \mathrm{m}$, if assumed circular) were neglected because they were not clearly distinguishable from image noise. Small emitting spots are more frequent than large ones. The number of spots is inversely proportional to their area, as indicated by the hyperbolic curve (continuous line) obtained by the fitting of the data in Fig. $6(\mathrm{R}=0.9797)$. The total emitting area was found to be about $3 \%\left(1860 \mu \mathrm{m}^{2}\right)$ of the total area of the micrograph $\left(64160 \mu \mathrm{m}^{2}\right)$. This percentage and the depth of the emitting channels $(\sim 10 \mu \mathrm{m}$ from the profiles in Fig. 5a) allowed to deduce the emitting volume and, in turn, the content of F-HA per lens (few tenths of microgram), taking into consideration the F-HA solution concentration. Therefore, fluorescence confocal microscopy is found to be very sensitive, down to such a low content of F-HA per lens, which could not be detected by simple optical absorption measurements performed on the lens. Indeed, the maximum absorption of a F-HA solution with concentration $0.2 \% \mathrm{~W} / \mathrm{v}$ taken with a $10 \mathrm{~mm}$ long cuvette was measured at $496 \mathrm{~nm}$ to be approximately 2 absorbance units. The thickness of one lens (approximated to be $100 \mu \mathrm{m})$ and its hydration $(\sim 50 \%)$ indicate that the absorbance attributable to the internalized F-HA solution cannot intrinsically be larger than about 1/200 of the measured absorbance of the solution, i.e. 0.01 , which is close to the limit of sensitivity of common spectrophotometers. If 0.01 absorbance is considered to be the experimental sensitivity limit, the 
measurement of the absorption spectrum of the lens is likely to be sensitive only to lenses which are completely hydrated by the F-HA solution. However, in our case, this absorbance value is expected to be further reduced by the shape and size of the lens pores, which prevent the F-HA internalization in the core of the lens. For this reasons, optical absorption on the lens was found to be not enough sensitive to provide experimental evidence of the F-HA internalization (the spectra are here omitted).

After wear, the scenario was found to change. After eight hours of wear, some differences were already detectable compared to the unworn lenses, as can be seen in Fig. 7a. The differences became definitively evident after one week of daily wear, when a deep change in morphology was observed, as observed in the SEM micrograph of the lens surface reported in Fig. $7 \mathrm{~b}$. The surface is characterized by a granular bumpy morphology. The appearance resembles one of the possible morphologies observed by AFM on worn lenses after one-day wear, as reported elsewhere. ${ }^{[27,28]}$ Both by SEM (present work) and by AFM, no large structures deposited on the lens surface were detected after one week. After two weeks, the scenario completely changed and Fig. 8 shows two representative SEM micrographs. A new corrugated morphology appeared, with crests and a more jagged structure. The micrographs suggest the formation of bulges or regions of swelling, which could be attributed to a relaxation of the polymeric network close to the surface. These bulges could be filled by a solution, as can be seen in Fig. 9, where the fluorescence micrograph of a lens after two weeks of wear and after F-HA loading is reported. No defined channels are observed, but large emitting areas. The penetration extent of F-HA across the thickness of the lens is shown at the bottom and right sides and it is comparable to the penetration extent in new lenses (Fig. 5).

\section{Conclusions}

SEM and fluorescence confocal microscopy allowed obtaining information on the structure and loading capability of siloxane-hydrogel contact lenses, in terms of (i) distribution and filling by a 
solute of the pores of the lens and (ii) modifications of the lens morphology and uptake as a consequence of wear. The surface of the siloxane-hydrogel material was found to show relatively large pores, with diameters of the order of magnitude of the micrometer or larger. After the F-HA loading, the filling of the pores was found to be almost complete and a new branched morphology was observed with a general thickening of the main walls of the pores. F-HA was found to fill the pores reaching a variable penetration depth from few micrometers to tens of $\mu \mathrm{m}$, to be compared to the thickness of the lens equal or larger than $90 \mu \mathrm{m}$. This variation was attributed to differences in the depth of the pores in different portions of the same lens and by a lens to another. Approximately, the content of F-HA per lens was evaluated to be of the order of few tenths of microgram. This value is non negligible, but it is limited by the depth of the siloxane-hydrogel channels, thus being far from the possible F-HA uptake of a lens with a deeper extended porosity. After wear, a different scenario was found. A progressive modification was observed. The longest time of wear of the present study (two weeks) produced a buggy morphology with regions of swelling on the surface, which could be filled by a solute. The modification was attributed to the relaxation of the polymeric network close to the surface. Preliminary results obtained on other siloxane-hydrogel contact lenses both before and after wear indicated similar scenarios (see Supporting Information).

\section{Acknowledgments}

We thank Silvia Trabattoni for helpful discussion and Alessandro Filippo for his support. 


\section{References}

[1] Karlgard CCS, Wong NS, Jones LW, Moresoli C. In vitro uptake and release studies of ocular pharmaceutical agents by silicon-containing and p-HEMA hydrogel contact lens materials. International Journal of Pharmaceutics. 2003; 257:141-151.

[2] Hiratani H, Fujiwara A, Tamiya Y, Mizutani Y, Alvarez-Lorenzo C. Ocular release of timolol from molecularly imprinted soft contact lenses. Biomaterials. 2005; 26: 1293-1298.

[3] Xinming L., Yingde C, Lloyd AW,Mikhalovsky SV, Sandeman SR, Howel CA, Liewen L. Polymeric hydrogels for novel contact lens-based ophthalmic drug delivery systems: A review. Contact Lens and Anterior Eye. 2008; 31: 57-64.

[4] Kogan G, Solte's L, Stern R, Gemeiner P. Hyaluronic acid: a natural biopolymer with a broad range of biomedical and industrial applications. Biotechnol Lett. 2007;29:17-25.

[5] Polack FM, McNiece MT. The treatment of dry eyes with Na hyaluronate-Healon. Cornea $1982 ; 1: 133-136$.

[6] Limberg MB, McCaa C, Kissling GE, Kaufman HE. Topical application of hyaluronic acid and chondroitin sulfate in the treatment of dry eyes. Am J Ophthalmol. 1987;103:194-197.

[7] Hamano T, Horimoto K, Lee M, Komemushi S. Sodium hyaluronate eye drops enhance tear film stability. Jpn J Ophthalmol. 1996;40:60-65.

[8] Yokoi N, Komuro A, Nishida K, Kinoshita S. Effectiveness of hyaluronan on corneal epithelial barrier function in dry eye. Brit J Ophthalmol. 1997;81:533-536.

[9] Aragona P, Papa V, Micali A, Santocono M, Milazzo G. Long term treatment with sodium hyaluronate-containing artificial tear reduces ocular surface damage in patients with dry eye. Brit J Ophthalmol. 2002;86:181-184.

[10] Balazs EA. Viscoelastic properties of hyaluronan and its therapeutic use. In: Garg HG, Hales CA, editors. Chemistry and biology of hyaluronan. Amsterdam: Elsevier. 2004:415. 
[11] Falcone SJ, Palmeri D, Berg RA. Biomedical applications of hyaluronic acid. Polysaccharides Drug Deliv Pharm Appl (ACS Symp Ser) 2006;155-174.

[12] Johnson ME, Murphy PJ, Boulton M. Effectiveness of sodium hyaluronate eyedrops in the treatment of dry eye. Graefes Arch Clin Exp Ophthalmol 2006; 244:109-112.

[13] Safilens Srl, International Application Published under the Patent Cooperation Treaty, International Publication Number WO 2006/085351 A1, 17 August 2006.

[14] Weeks A, Subbaraman LN, Jones L, Sheardown H. Physical entrapment of hyaluronic acid during synthesis results in extended release from model hydrogel and silicone hydrogel contact lens materials. Eye \& Contact lens 2013; 39: 179.

[15] Van Beek M, Jones L, Sheardown H. Hyaluronic acid containing hydrogels for the reduction of protein adsorption. Biomater 2008; 29: 780.

[16] Van Beek M, Weeks A, Jones L, Sheardown H. Immobilized hyaluronic acid containing model silicone hydrogels reduce protein adsorption. J Biomater. Sci. Polym. Ed. 2008; 19: 1425.

[17] Leonardi A, Decastello N, Brun P, Violato D, Fregona I. Release of Hyaluronic Acid From Filcon1b Contact Lens Increases Tolerability and Performance. ARVO's 2007 Annual Meeting, May 6-10 2007, Fort Lauderdale, Florida.

[18] Fagnola M, Pagani MP, Maffioletti S, Tavazzi S, Papagni A. Hyaluronic acid in hydrophilic contact lenses: Spectroscopic investigation of the content and release in solution. Cont Lens Anterior Eye. 2009;32:108-112.

[19] Hiratani H, Alvarez-Lorenzo C. Timolol uptake and release by imprinted soft contact lenses made of N,N-diethylacrylamide and methacrylic acid. J Controll Rel 2002; 83: 223.

[20] Ganji F, Vasheghani-Farahani S, Vasheghani-Farahani E. Theoretical Description of Hydrogel Swelling: A Review. iranian Pol J 2010; 19: 375.

[21] Kim SW, Bae YH, Okano T. Hydrogels: swelling, drug loading, and release. Pharm Res 1992; 9: 283. 
[22] M.D. Merindano, M. Canals, C. Saona, J. Potau, J. Costa, Observation of deposits on disposable contact lenses by bio- light- and scanning electron microscopy, Contact Lens and Anterior Eye, 1998, 21:55-59.

[23] F. Yanez, J. Gomez-Amoza, B. Magarinos, A. Concheiro, C. Alvarez-Lorenzo, Hydrogels porosity and bacteria penetration: Where is the pore size threshold?, Journal of Membrane Science , 2010, 365:248-255.

[24] J. M. Gonzalez-Meijome, A. Lopez-Alemany, J. B. Almenida, M. A. Parafita, M. F. Refojos, Microscopic observations of superficial ultrastructure of unworn siloxane-hydrogel contact lenses by cryo-scanning electron microscopy, Wiley Periodicals, 2005, 419-423.

[25] V. Guryca, R. Hobzova, M. Pradny, J. Sirc, J. Michalek, Surface morphology of contact lenses probed with microscopy technique, Contact Lens \& Anterior Eye, 2007, 30:215-222.

[26] Lowman AM, Smart $\quad$ Pharmaceuticals, http://wwwgateway.vpr.drexel.edu/files/NewEh/htmls/lowman.pdf, 2008.

[27] Bettuelli M, Trabattoni S, Fagnola M, Tavazzi S, Introzzi L, Farris S. Surface properties and wear performances of siloxane-hydrogel contact lenses. J Biomed Mater Res B 2013; 101B: $1585-1593$.

[28] Goldberg EP, Bhatia S, Enns JB. Hydrogel contact lens-corneal interactions: a new mechanism for deposit formation and corneal injury. CLAO J. 1997;23:243-248. 


\section{FIGURE CAPTIONS}

Fig. 1. Surface SEM micrographs of a F-HA-free contact lens taken on two different areas (a and b) of the lens.

Fig. 2. Surface SEM micrographs of two F-HA-loaded contact lenses ( $a$ and b).

Fig. 3. Surface micrographs of of two F-HA-loaded contact lenses ( $a$ and $b$ ).

Fig. 4. Depth profitle micrograph of a F-HA-loaded contact lens.

Fig. 5.Fluorescence confocal micrographs taken from the surface of two F-HA-loaded contact lenses (main micrographs) and depth profiles along the grey lines (right and bottom).

Fig. 6.Statistical frequency (bars) of the area of the fluorescence spots in the micrograph of Fig. 5a and hyperbolic curve obtained from the data fitting $(\mathrm{R}=0.9797)$.

Fig. 7. Surface micrographs of (a) a F-HA-loaded contact lens after eight hours of wear and (b) a FHA-loaded contact lens after one week of daily wear.

Fig. 8. Surface micrographs of two different F-HA-loaded contact lenses (a and b) after two weeks of daily wear.

Fig. 9. Fluorescence confocal micrograph taken from the surface (main micrographs) of a F-HAloaded contact lens after two weeks of daily wear and depth profiles along the grey lines (right and bottom). 


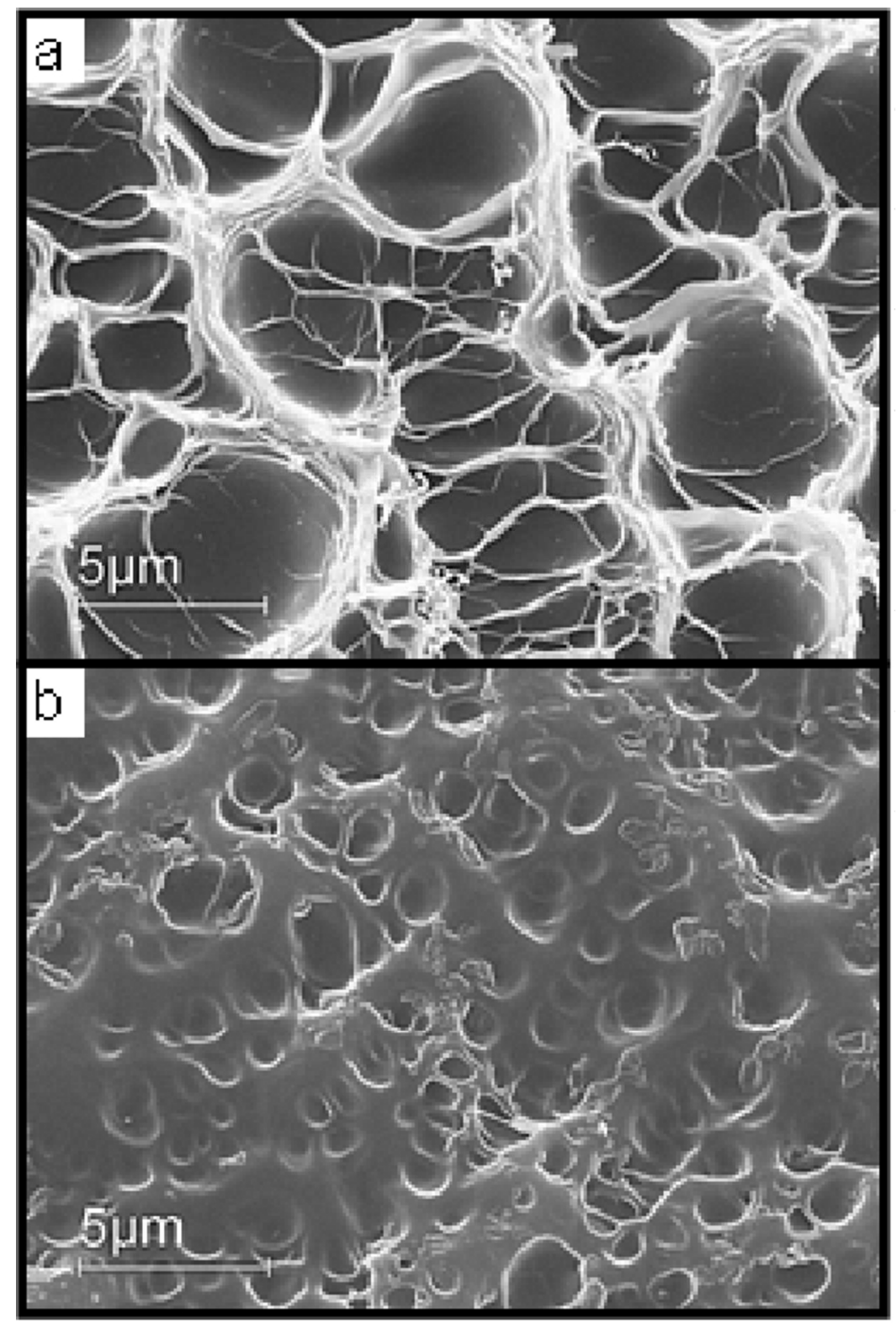

Fig. 1. Surface SEM micrographs of a F-HA-free contact lens taken on two different areas ( $a$ and $b)$ of the lens. 


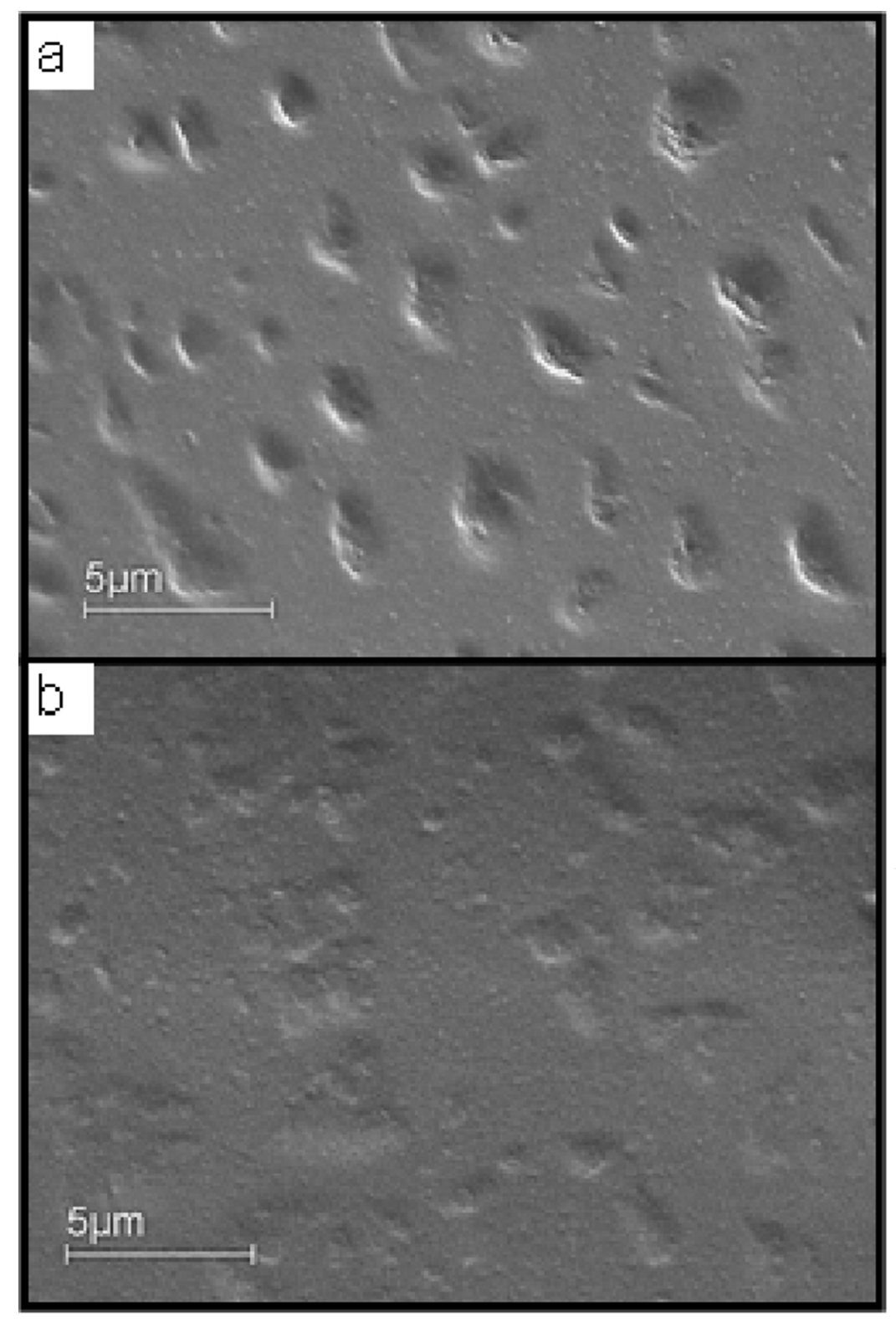

Fig. 2. Surface SEM micrographs of two F-HA-loaded contact lenses ( $a$ and $b$ ). $294 \times 439 \mathrm{~mm}(96 \times 96 \mathrm{DPI})$ 


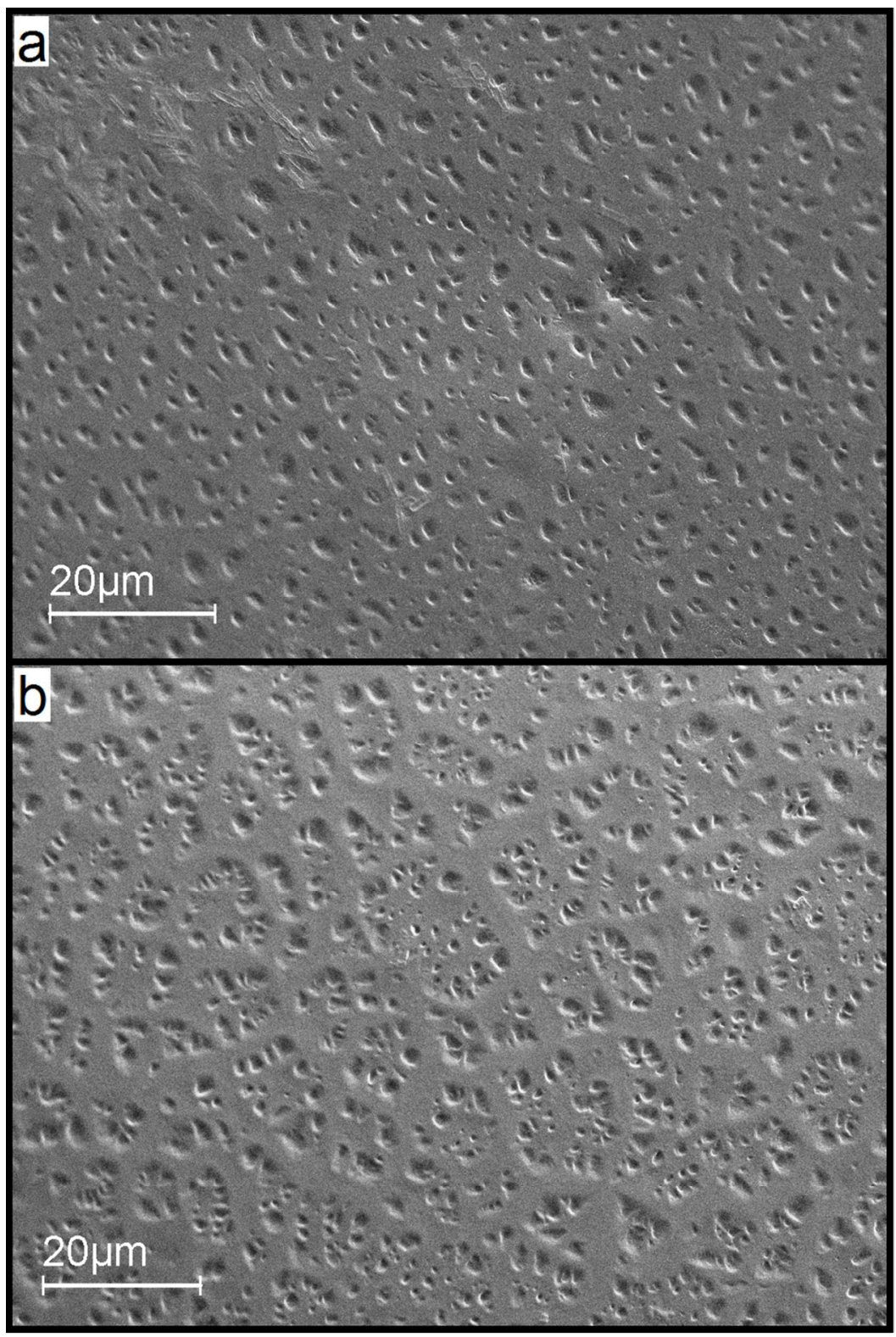

Fig. 3. Surface micrographs of of two F-HA-loaded contact lenses ( $a$ and $b$ ). $297 \times 443 \mathrm{~mm}(96 \times 96$ DPI)

John Wiley \& Sons, Inc. 


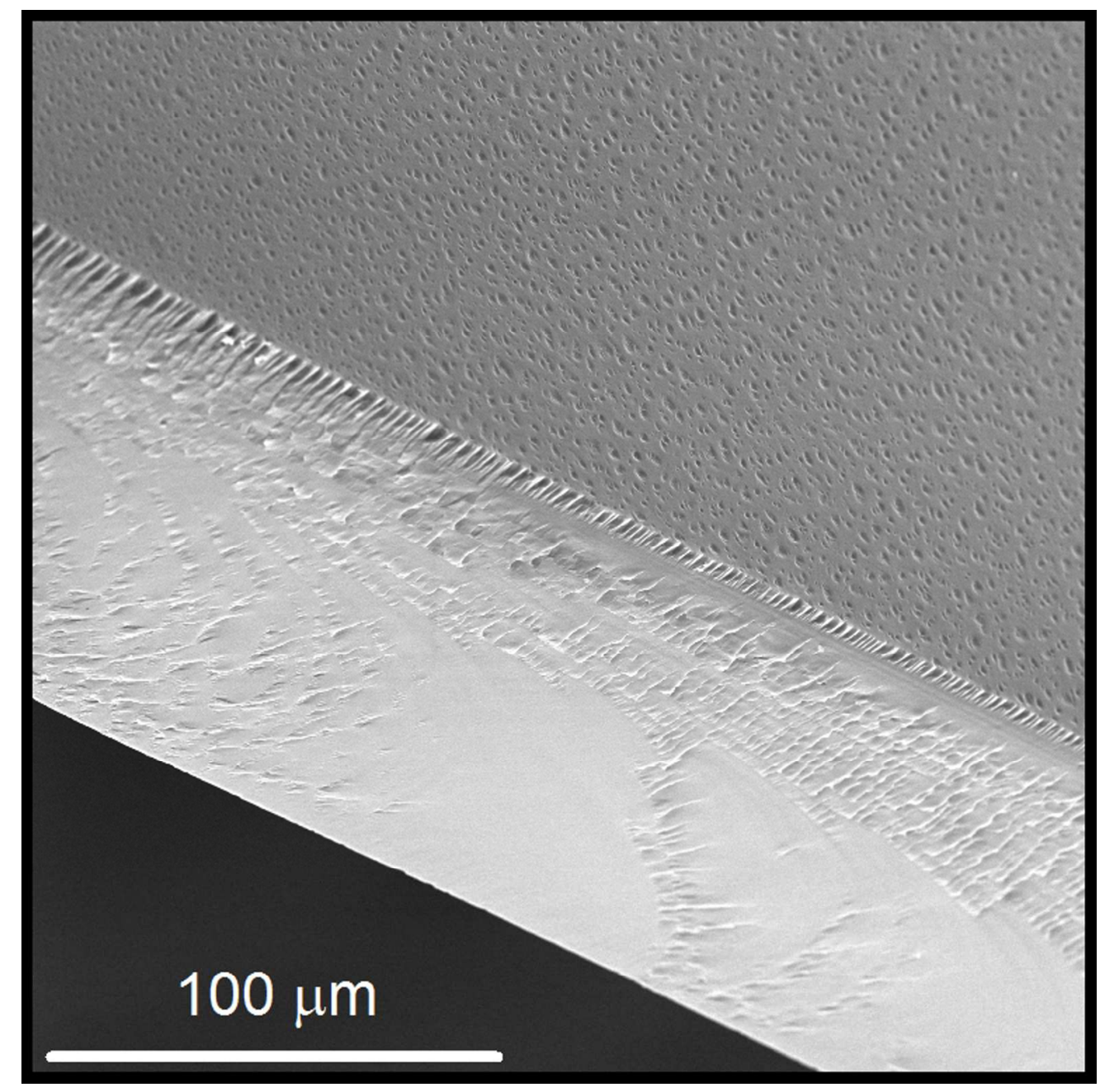

Fig. 4. Depth profitle micrograph of a F-HA-loaded contact lens. $394 \times 393 \mathrm{~mm}(96 \times 96 \mathrm{DPI})$ 


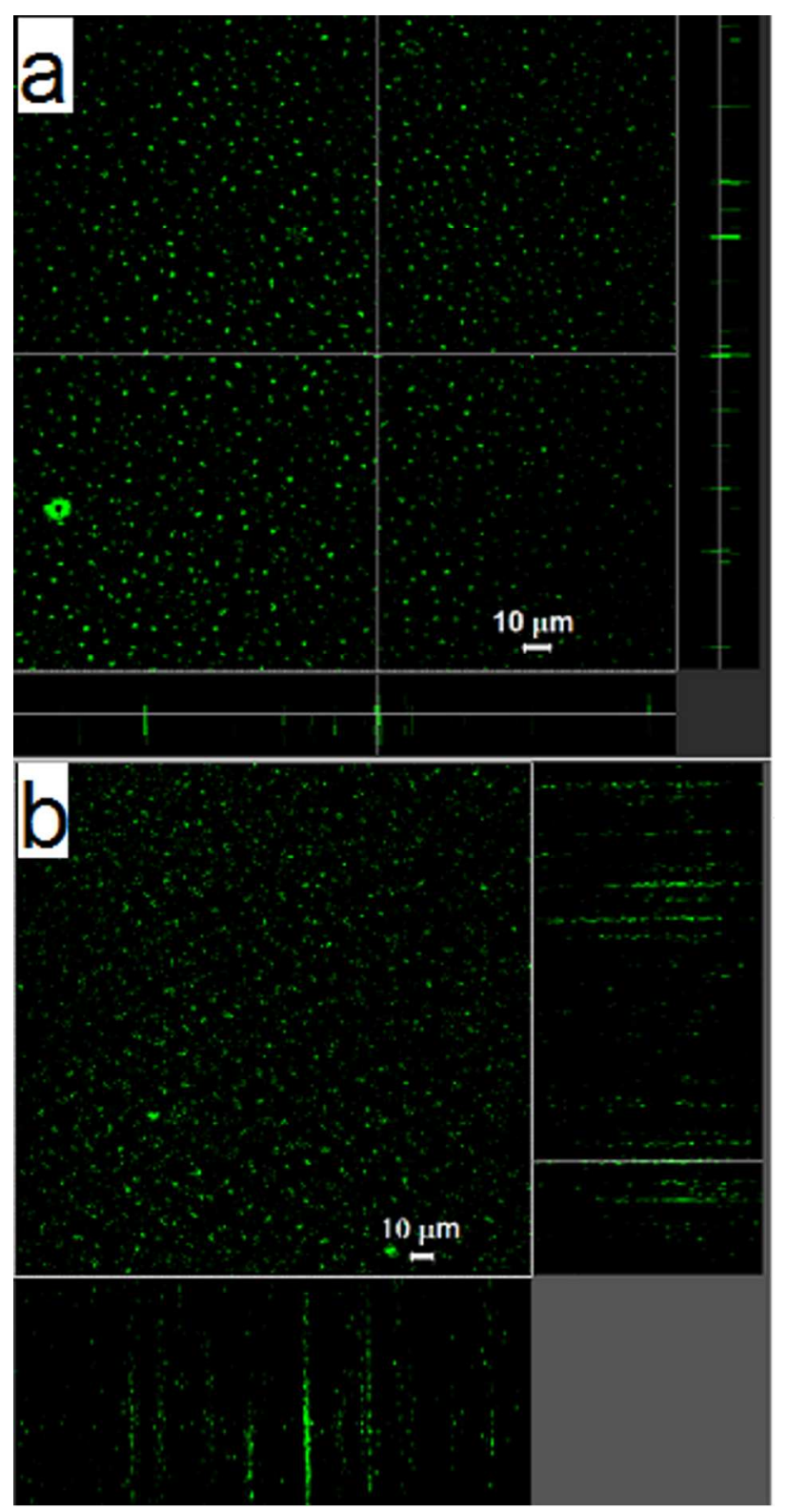

Fig. 5.Fluorescence confocal micrographs taken from the surface of two F-HA-loaded contact lenses (main micrographs) and depth profiles along the grey lines (right and bottom). $273 \times 535 \mathrm{~mm}(96 \times 96 \mathrm{DPI})$ 


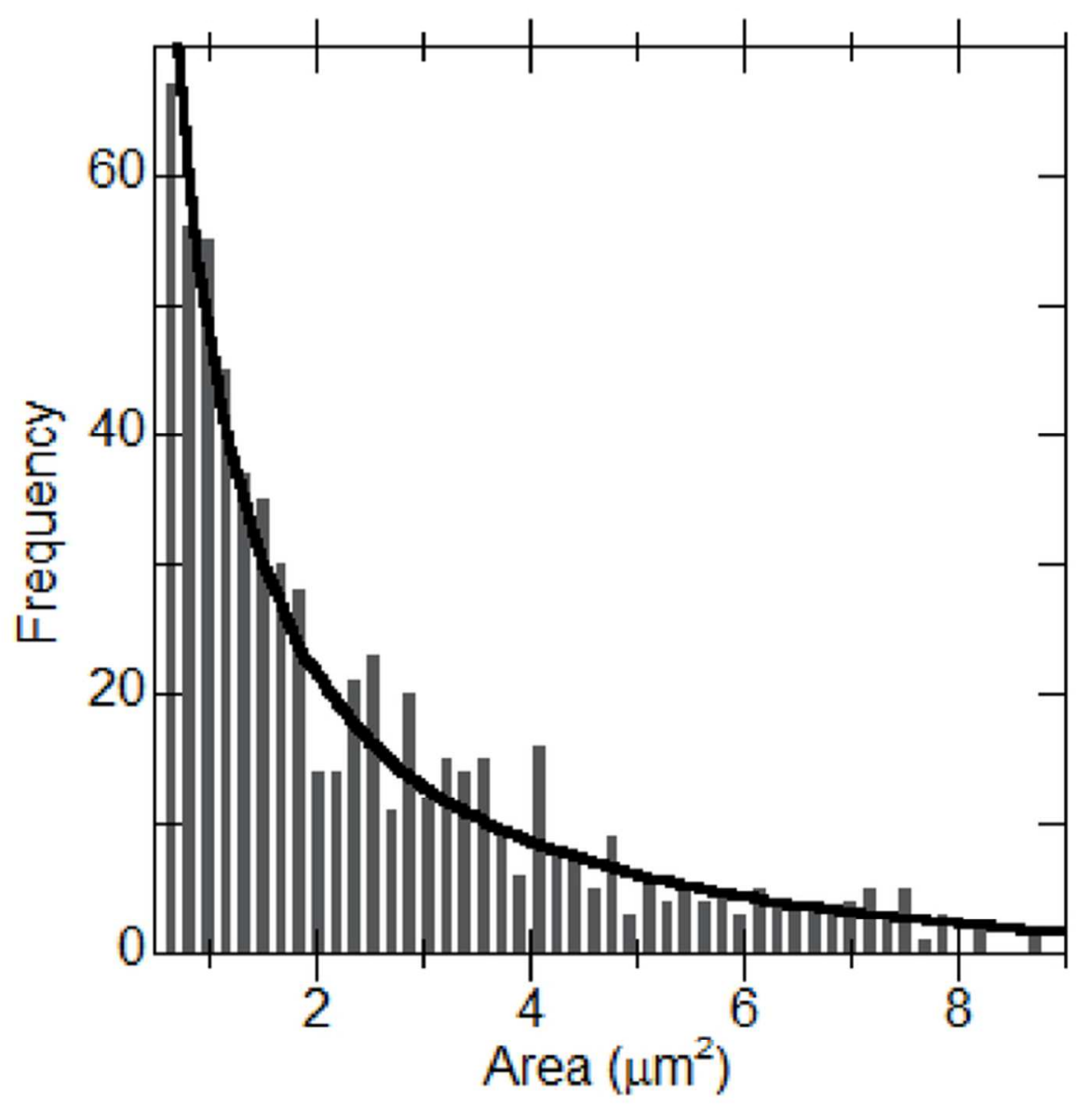

Fig. 6.Statistical frequency (bars) of the area of the fluorescence spots in the micrograph of Fig. 5a and hyperbolic curve obtained from the data fitting $(R=0.9797)$. $67 \times 67 \mathrm{~mm}(300 \times 300 \mathrm{DPI})$ 


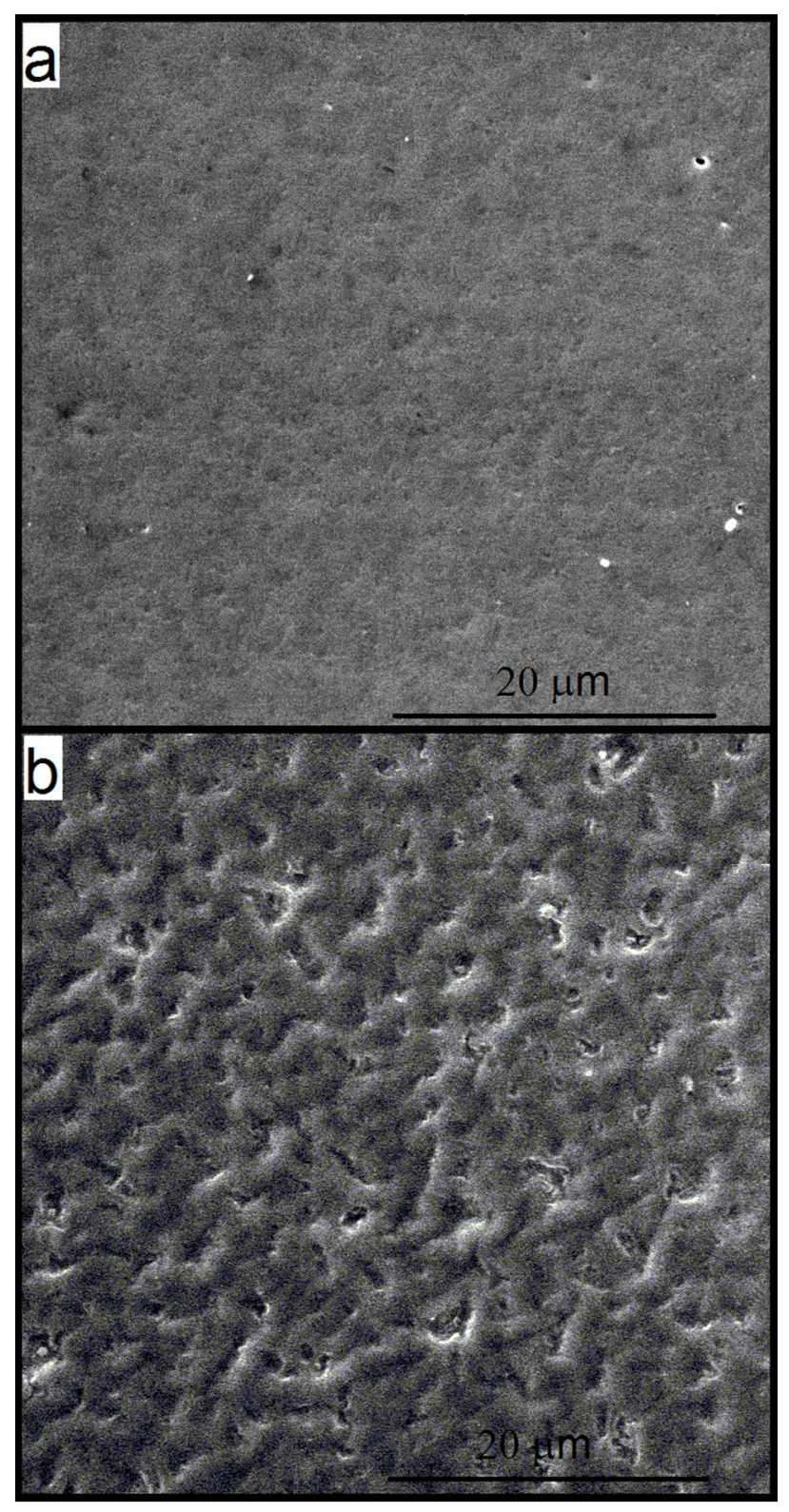

Fig. 7. Surface micrographs of (a) a F-HA-loaded contact lens after eight hours of wear and (b) a F-HAloaded contact lens after one week of daily wear. $260 \times 508 \mathrm{~mm}(96 \times 96 \mathrm{DPI})$ 


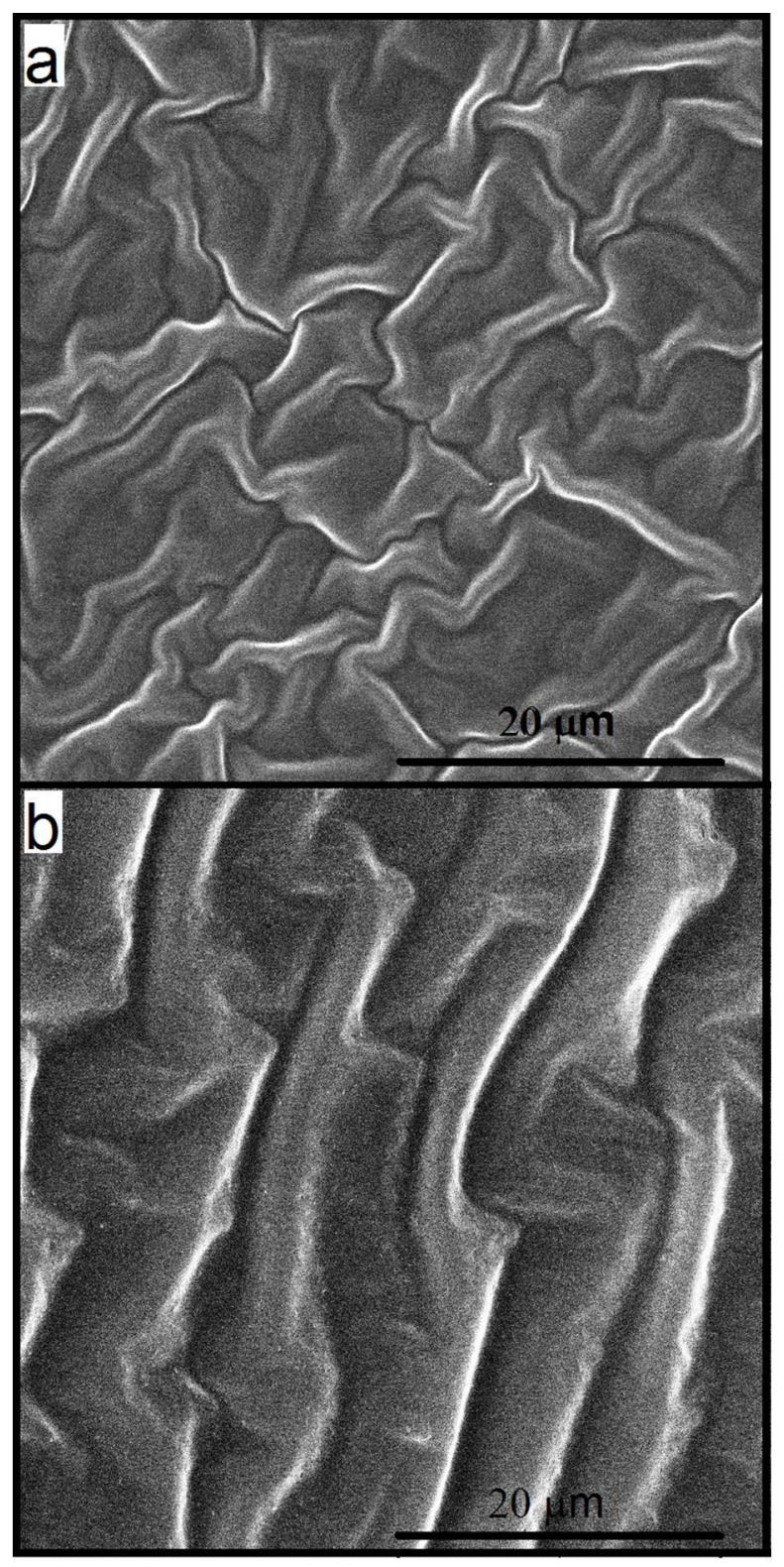

Fig. 8. Surface micrographs of two different F-HA-loaded contact lenses ( $a$ and $b$ ) after two weeks of daily wear. 


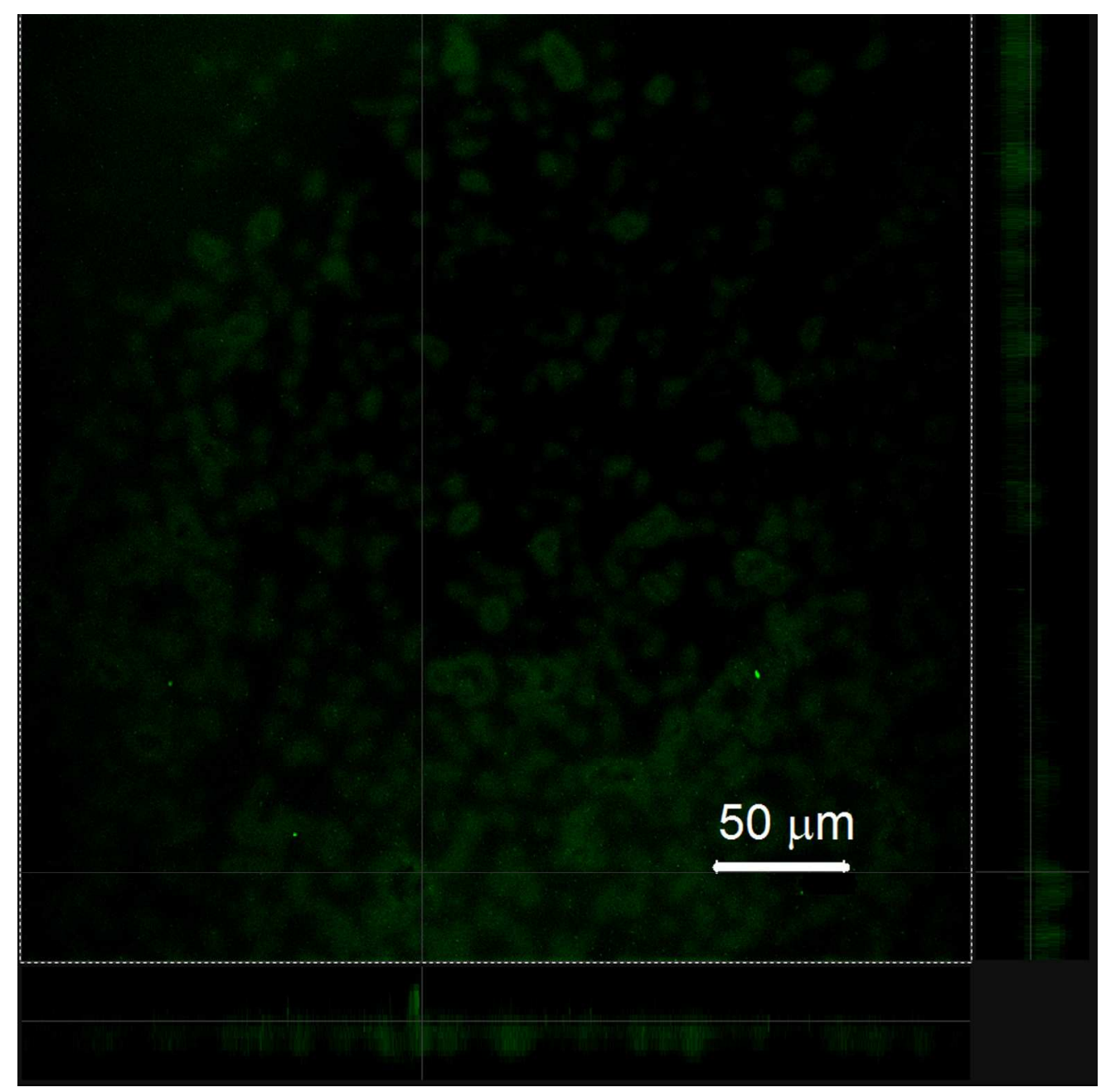

Fig. 9. Fluorescence confocal micrograph taken from the surface (main micrographs) of a F-HA-loaded contact lens after two weeks of daily wear and depth profiles along the grey lines (right and bottom). $439 \times 436 \mathrm{~mm}(96 \times 96 \mathrm{DPI})$ 